\title{
Contrôle et traitement des eaux et des effluents par techniques UV
}

\author{
O. Thomas, B. Roig et M.-F. Pouet
}

\author{
Laboratoire Génie de l'Environnement Industriel, École des Mines d'Alès, \\ 6 avenue de Clavières, 30319 Alès cedex, France
}

\begin{abstract}
Résumé : L'usage de sources UV dans les technologies environnementales est particulièrement répandu pour le contrôle de la qualité des eaux et des effluents et leur traitement. Dans les deux cas, le domaine couvert est de 180 à $350 \mathrm{~nm}$ et les lampes utilisées, très classiques, sont du type $\mathrm{Hg}$ basse pression ou deutérium. Différentes applications sont présentées comme la mesure de la qualité des milieux (spectrophotométrie directe ou indirecte) ou le traitement par procédés de photooxydation avancée.
\end{abstract}

\section{APPLICATIONS DE L'UV POUR LA MESURE ET LE TRAITEMENT DE LA POLLUTION DES EAUX}

L'évolution des contraintes réglementaires et des normes dans le domaine de l'environnement, en particulier celles destinées à la réduction des pollutions aqueuses, s'accompagne de développements technologiques basés notamment sur l'utilisation de sources UV.

\subsection{Mesure de qualité}

Depuis quelques années, des systèmes spectrophotométriques de mesure de la qualité des eaux et des effluents, basés sur l'utilisation de méthodes optimales d'exploitation des spectres UV (Fig. 1), permettent d'obtenir aisément des données sur la qualité des milieux surveillés [1-3]. Les paramètres concernés sont représentatifs de la pollution éventuelle (pollution globale, azotée, ou liée à des composés organiques spécifiques) mais aussi des caractéristiques d'évolution de ces milieux, ce qui rend possible la détection d'incidents et la prévision de leur impact. Des analyseurs de terrain (portable ou en ligne) sont commercialisés depuis près de deux ans.
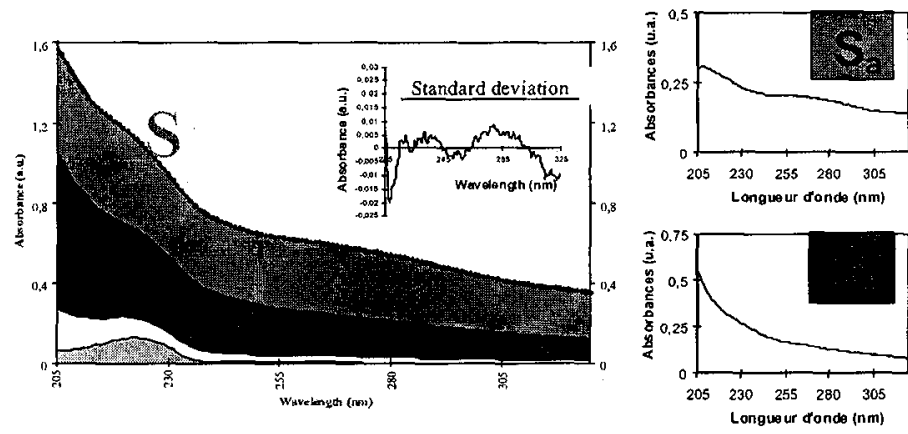

Exemple : pour chaque longueur d'onde $\lambda$ :

$$
\mathrm{S}=1.73 * \mathrm{~S} \quad \mathrm{a}+1.41 * \mathrm{~S}_{\mathrm{b}}+0.25 * \mathrm{~S}_{\mathrm{c}}+0.00 * \mathrm{~S}_{\mathrm{d}}+0.11 * \mathrm{~S} \quad \mathrm{e}^{+\mathrm{r}}
$$
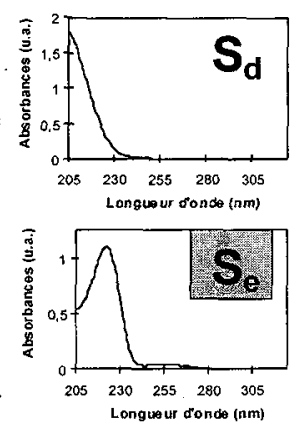

Figure 1 : Procédure de déconvolution semi-déterministe d'exploitation de spectre UV [2]

Dans certains cas, la mise en œuvre d'un couplage UV/UV (photodégradation de l'échantillon suivi d'une détection UV) est nécessaire [4,5]. Cette approche originale et très récente permet une amélioration notable des procédures existantes. Dans ce cas, le type de lampe utilisée est une lampe à $\mathrm{Hg}$ basse pression et le quartz de qualité Suprasil (B) (Fig. 2). Ce système de prétraitement simple permet notamment une analyse rapide et simple des différentes formes de l'azote dans les eaux. 

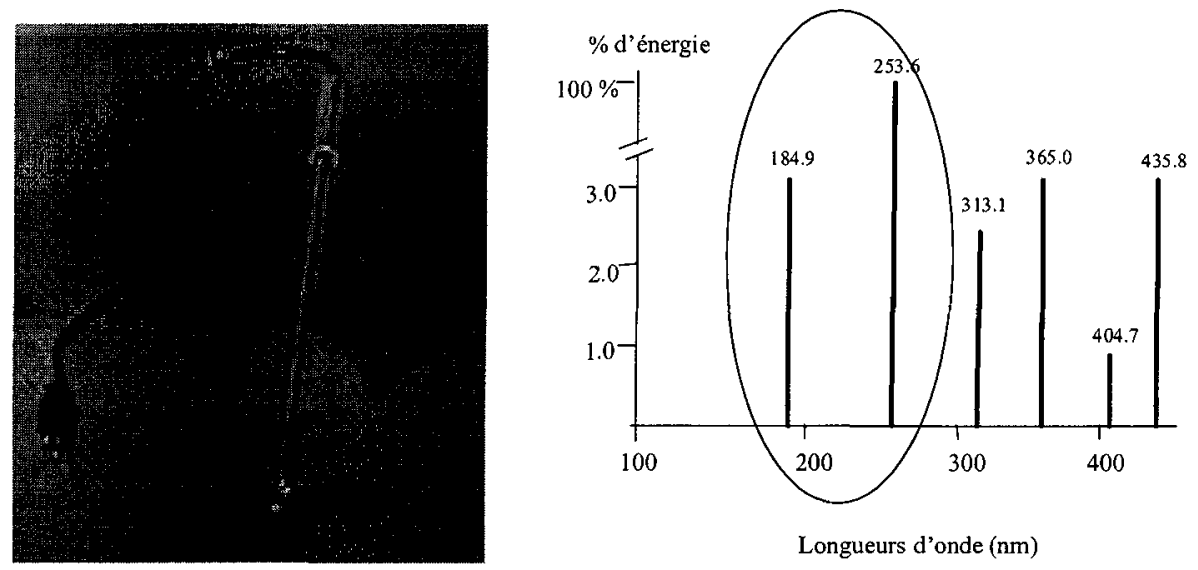

Figure 2 : Lampe Pen Ray (UVP), puissance électrique $45 \mathrm{~W}$, nominale $6 \mathrm{~W}$ à $253,6 \mathrm{~nm}$ et $0,2 \mathrm{~W}$ à $184,9 \mathrm{~nm}$.

Ces méthodes, alternatives au sens de la norme XPT 90-210 permettent non seulement de vérifier la qualité des eaux d'un point de vue réglementaire mais permettent également de contrôler, et donc d'optimiser, toute procédé de traitement. Les informations qualitatives fournies par ces techniques (photodégradabilité, variabilité, conservation de quantité et/ou qualité) intégrées dans des outils d'aide à la décision permettent aussi de mieux maîtriser les flux polluants, de les réduire tout en orientant l'utilisateur vers les meilleures filières de traitement [8].

\subsection{Traitement des effluents par POA}

Malgré les efforts entrepris pour la réduction des flux polluants, notamment à l'aide des systèmes précédents, le traitement des effluents demeure indispensable. Pour certaines eaux résiduaires concentrées, de nouvelles techniques d'oxydation, dites procédés d'oxydation avancée (POA), peuvent être utilisées à la place de techniques lourdes comme l'incinération, économiquement et écologiquement peu acceptable. Ces techniques sont basées sur la photooxydation des effluents en associant un rayonnement UV «dur » (en dessous de $200 \mathrm{~nm}$ ) et un oxydant usuel (eau oxygénée par exemple) [6]. Les sources sont également des lampes $\mathrm{Hg}$ basse pression et le quartz utilisé de qualité Suprasilß. Les résultats en laboratoire sont souvent très satisfaisants : le couplage $\mathrm{UV} / \mathrm{H} 2 \mathrm{O} 2$, par exemple, permet notamment d'augmenter la biodégradabilité d'effluents industriels caractérisés par une forte pollution organique dure (DCO dure). La transposition industrielle permettra de vérifier prochainement l'intérêt de ces techniques alternatives.

Pour d'autres applications, plus simples, l'usage direct de rayonnement UV avec des sources plus communes ( $\mathrm{Hg}$ basse pression avec raie à $254 \mathrm{~nm}$ et quartz de qualité Infrasil@), associées ou non avec des oxydants comme l'ozone permet de «désinfecter » des milieux aqueux voir de détruire quelques molécules organiques facilement oxydables. Dans les deux applications précédentes (mesure et traitement), les systèmes développés sont simples, fiables et économiquement acceptables par rapport aux solutions existantes (lorsqu'elles existent) [7].

\section{UN BESOIN DE NOUVELLES SOURCES UV}

Du point de vue des sources UV utilisées, deux types de lampes sont retenues à l'heure actuelle, les critères de choix résultant d'un compromis technico-économique. Les techniques spectrophotométriques utilisent des lampes Deutérium, classiques ou pulsées, qui délivrent un spectre d'émission continu dans le domaine 190-350 $\mathrm{nm}$. Ces sources évoluent constamment sous l'impulsion des fabricants tout en restant relativement onéreuses et « énergétivores ». Les techniques de traitement utilisent des lampes à vapeur de mercure basse pression, préférées aux lampes moyenne pression par leur coût, leur facilité de mise en œuvre et leur durée. Cependant, des problèmes techniques subsistent dans le choix des sources disponibles et des matériaux accessoires (quartz) pour le traitement par photooxydation des effluents. L'absence de procédures simples pour qualifier ces éléments (actinomètres, radiomètres, ...) couplée au manque de sérieux des fournisseurs, constitue à l'heure actuelle un frein pour le développement de ces techniques. Par ailleurs, l'interdiction programmée à plus ou moins longue échéance du mercure obligera à l'usage d'autres type de lampes. 
D'autres sources potentielles comme les lampes à excimères ou les lasers ne semblent pas (à l'heure actuelle) éligibles du point de vue économique. Le besoin de disposer d'une source économique, de mise en œuvre peu contraignante, émettant vers $180 \mathrm{~nm}$ est donc évident.

\section{Remerciements}

Les auteurs remercient D. Constant (Secomam) pour les développements instrumentaux.

\section{Références}

1. O. Thomas, F. Theraulaz, M. Domeizel, C. Massiani, Environ. Technol., 14, 1187-1192, 1993

2. O. Thomas, F. Theraulaz, C. Agnel, S. Suryani, Envir. Technol., vol. 17, pp. 251-261, 1996

3. O. Thomas, F. Theraulaz, Cerdà V., D. Constant, P. Quevauviller, Trends in analytical chemistry, vol. $16, n^{\circ} 7$, pp. $419-424,1997$

4. B. Roig, C. Gonzalez, O. Thomas, Analytical Chimica Acta 389, pp. 267-274, 1999

5. B. Roig, C. Gonzalez, O. Thomas, Talanta 50, pp. 751-758, (1999)

6. L. Castillo, A. Sillet, J. Roussy, J-R. Degorce-Dumas, O. Thomas, Water Science and Technology, acceptée

7. M-F. Pouet, O. Thomas, Bo N. Jacobsen, A. Lynggaard-Jensen, P. Quevauviller, Talanta 50, pp. 759 $762(1999)$

8. Muret C., Pouet M.-F., Touraud E. and Thomas O. (2000) Water Science and Technology, 42 (5-6) 47-53. 